from a superposition of the jet effect on a general field, the fluctuations arise mainly from minor fluctuations of the electro-jet itself.

The rapid change in daily range with latitude indicates that the equatorial electro-jet must be very narrow, probably about $2-3^{\circ}(200-300 \mathrm{~km}$.) wide. This is supported by the anomalously large diurnal variation in the vertical component $Z$ of the magnetic field at Ibadan. The mean quiet-day range in $Z$ varies seasonally from about $40 \gamma$ to $80 \gamma$, and is fairly constantly about $0 \cdot 6$ of the daily range of $H$. This indicates an origin in a current system the centre of gravity' of which is well to the north. Ibadan appears to be not directly under the electro-jet, but sufficiently close to it for its effects to predominate in the daily variation.

A parallel investigation of the variation of $Z$ with latitude is being undertaken shortly, and it is hoped that this will give more detailed information on the location and extent of the equatorial electro-jet.

Full details will be published shortly.

N. S. ALEXANDER

C. A. ONWUMECHILLI

Physics Department,

University College,

Ibadan.

May 13.

'Chapman, S., and Bartels, J., "Geomagnetism", 242 (1940).

\section{A Frequency Comparator using Electron and Proton Resonance in a Common Magnetic Field}

HIGH microwave frequencies may, with advantage, be measured by utilizing electron and proton paramagnetic resonances in a common magnetic field. For this purpose the free radical diphenyl trinitrophenyl hydrazyl, which has one of the strongest and narrowest lines yet reported ${ }^{1}$, is suitable for the electron resonance sample. The protons in water containing a trace of a paramagnetic salt provide the most suitable substance for the proton resonance.

The method of frequency measurement at, say, $35,000 \mathrm{Mc} / \mathrm{s}$. is as follows. The magnetic field is varied until a signal is observed from the electron resonance. This will be at about 12,000 gauss. This field is then measured by means of a proton resonance fluxmeter. If the common magnetic field is swept through a small range in synchronism with the $X$-deflexion of a cathode ray tube, it is possible to superimpose the proton and electron signal with considerable precision despite small instabilities in the magnetic field.

When this has been done the ratio of the two frequencies depends on the ratio of the two gyromagnetic ratios $\gamma_{e} / \gamma_{p} \bumpeq 658 \cdot 5$. In this way a conventional high grade signal generator employing quartz crystal calibration points may be used to read the lower frequency; and by means of the multiplying factor $658 \cdot 5$, converted to the microwave frequency. Alternatively a quartz harmonic generator may be used, of fundamental frequency $10^{n} / 658 \cdot 5$ Mc./s., and this will provide markers at $10^{n} \mathrm{Mc} / \mathrm{s}$. intervals, where $n$ is a convenient integer.

The accuracy of the system has yet to be more fully investigated, but it would appear that the most fundamental limitation is the rather wide line of polycrystalline hydrazyl. This has a width of
$8 \mathrm{Mc} / \mathrm{s}$. so the setting accuracy would be of the order of $0.8 \mathrm{Mc} / \mathrm{s}$. However, there may be better materials available, such as aminophenyl-aminium perchlorate, which is reported ${ }^{2}$ as having a line width of about $1 \mathrm{Mc} / \mathrm{s}$. Sodium in ammonia ${ }^{3}$ conld also be used but has practical difficulties. Its line width is reported as about $0.2 \mathrm{Mc}$./s. Another very practical limitation is the uniformity of the magnetic field required to render the field common to both specimens in space (or the stability required for the exchange to take place in time). The most promising method would appear to be to exchange the relative positions of electron and proton sample and take the mean of two or more observations. It is expected that the accuracy to which $\gamma_{e} / \gamma_{p}$ is known will improve as work proceeds.

This communication is published with the permission of the Admiralty.

Admiralty Signal and Radar Fistablishment, Portsdown, Cosham,

Portsmouth, Hants. March 4.

'Phys. Rev., 75, 1614 (1949).

${ }^{2}$ Phys. Rev., 73, 679 (1948)

${ }^{3}$ Phys. Rev., 81, 282 (1951).

\section{Use of 'Nobecutane' in attaching Skin Electrodes}

IN routine physiological experimentation, various types of electrodes are used attached to the surface of the body. These are commonly attached by rubber bands, strips of adhesive plaster or some kind of cement. At the R.A.F. Institute of Aviation Medicine, three types of electrode are being used which require a special method of attachment.

(1) For routine electrocardiograms in the acceleration research department, small silver disks $1.5 \mathrm{~cm}$. in diameter are used attached to the front of the chest for the $V$-leads and to the back for tho Duchosal and Sulzer method in vector cardiography. Contact with the skin is limited to the circumference of the disk, the centre position being recessed and containing electrode jelly. These electrodes and their leads are subjected to fairly severe strain when the subject is having accelerations of up to $7 G$ applied. An adhesive was required which would hold the electrode firmly in position and yet allow movement of the part and withstand a fair pulling strain.

(2) In the climatic research unit, experiments required the attachment of heat-flow disks and thermocouples to the skin which would be submerged in water, thus a strong adhesive with waterproof qualities was essential. After trying several methods of attachment a trial using 'Nobecutane' was commenced.

For the attachment of electrocardiographic electrodes, 'Nobecutane' proved highly satisfactory. The electrodes were quickly applied and the time spent in preparing the subject was cut down considerably. The breaking strain of a film of 'Nobecutane' was tested by attaching an electrode to the volar aspect of the forearm with a film of 'Nobecutane' 0.01 in. thick and adding weights until the electrode was detached. In five such tests, the weights required to pull off the electrode were $2 \cdot 0-2 \cdot 5 \mathrm{kgm}$.

In all, some fifty centrifuge runs have been carried out using electrodes stuck on with 'Nobecutane' with- 